Soon-Mo Jung, Mathematics Section, College of Science \& Technology

Hong-Ik University, 339-800 Chochiwon, South Korea. e-mail:

smjung@wow.hongik.ac.kr

\title{
ON THE HAUSDORFF MEASURE OF A CLASS OF SELF-SIMILAR SETS
}

\begin{abstract}
We develop a new combinatorial method to estimate Hausdorff measures of various self-similar sets. This method can be applied to the evaluation of Hausdorff measures which are induced by various Hausdorff functions including power functions. Moreover, a few examples for evaluations of the lower and upper bounds of Hausdorff measures of uniform Cantor sets are introduced.
\end{abstract}

\section{Introduction}

Throughout the paper, we use $\mathbb{N}_{0}, \mathbb{N}$ and $\mathbb{R}$ to denote the set of all nonnegative integers, of all positive integers and of all real numbers, respectively. $\mathbb{R}^{n}$ denotes $n$-dimensional Euclidean space, and $D$ will be some fixed closed subset of $\mathbb{R}^{n}$. By $d(A)$ we denote the diameter of any subset $A$ of $\mathbb{R}^{n}$, and the cardinal number of a set $C$ will be denoted by $\# C$.

A monotonically increasing function $h:[0, \infty) \rightarrow[0, \infty)$ is called a Hausdorff function if and only if $h(t)>0$ for $t>0, h(0)=0$ and $h$ is continuous from the right. It is well-known that every Hausdorff function $h$ induces a corresponding Hausdorff measure $\mu^{h}$ as follows

$$
\mu^{h}(C)=\lim _{\delta \rightarrow 0} \inf \sum_{i} h\left(d\left(U_{i}\right)\right),
$$

where we take the infimum over all $\delta$-coverings $\left\{U_{i}\right\}_{i}$ of $C$ (see [10]).

Key Words: Hausdorff function, Hausdorff measure, self-similar set

Mathematical Reviews subject classification: Primary 28A78; Secondary 28A12, 28A80

Received by the editors September 19, 1995 
A mapping $S: D \rightarrow D$ is called a similarity on $D$ if there is a constant $c$ $(0<c<1)$ such that $|S(x)-S(y)|=c|x-y|$ for all $x, y \in D$. The constant $c$ is called the (similarity) ratio of $S$.

Throughout the paper, suppose $m(>1)$ is a fixed integer. Let $\left\{S_{1}, \ldots, S_{m}\right\}$ be a family of similarities on $D$. We say that a subset $F$ of $D$ is self-similar under $S_{1}, \ldots, S_{m}$ if

$$
F=\bigcup_{i=1}^{m} S_{i}(F)
$$

Definition 1. A family $\left\{S_{1}, \ldots, S_{m}\right\}$ of similarities on $D$ is said to be disjoint if $S_{i}(D) \cap S_{j}(D)=\emptyset$ for all $i \neq j$.

Hutchinson [6] proved that for every disjoint family $\left\{S_{1}, \ldots, S_{m}\right\}$ of similarities on $D$ there exists a unique non-empty compact set which is self-similar under the $S_{i}$ 's. Many self-similar sets are well-known, e.g., Cantor sets, Cantor dusts, the Sierpiński gasket, the von Koch curve, etc.

For the time being, let $\left\{S_{1}, \ldots, S_{m}\right\}$ be any disjoint family of similarities on $D$ with the ratios $c_{1}, \ldots, c_{m}$, respectively. Let $\ell \in \mathbb{N}$ be fixed. By $\mathcal{I}_{\ell}$ we denote the family of all finite sequences $\left(a_{i}\right)_{i=1, \ldots, \ell}$ satisfying $a_{i} \in\{1, \ldots, m\}$, i.e.,

$$
\mathcal{I}_{\ell}=\left\{\left(a_{i}\right)_{i=1, \ldots, \ell}: a_{i} \in\{1, \ldots, m\}\right\}
$$

For every finite sequence $a=\left(a_{i}\right) \in \mathcal{I}_{\ell}$, let $S_{a}=S_{a_{1}} \circ S_{a_{2}} \circ \cdots \circ S_{a_{\ell}}$ with the convention

$$
S_{a}(x)=S_{a_{1}}\left(S_{a_{2}}\left(\cdots\left(S_{a_{\ell}}(x)\right) \cdots\right)\right) .
$$

We denote by $\mathcal{H}$ the class of all Hausdorff functions and by $\mathcal{H}_{m}^{c}$ the class of all Hausdorff functions satisfying

$$
h(c t)=\frac{1}{m} h(t)
$$

for all sufficiently small $t>0$.

In this paper, a new combinatorial method to estimate Hausdorff measures (which are induced not only by power functions but also by other Hausdorff functions different from power functions) of self-similar sets are investigated (see Theorem 8), and the method is applied to the estimation of Hausdorff measures of uniform Cantor sets. 


\section{Preliminaries}

Definition 2. Suppose $\left\{S_{1}, \ldots, S_{m}\right\}$ is a disjoint family of similarities on $D$. Let $C$ be an arbitrary subset of $\mathbb{R}^{n}$. For any non-negative integer $i$ let

$$
\alpha_{i}(C)=\lim _{\ell \rightarrow \infty} \frac{\#\left\{a \in \mathcal{I}_{i+\ell}: S_{a}(D) \subset C\right\}}{m^{\ell}} .
$$

Lemma 1. Let $\left\{S_{1}, \ldots, S_{m}\right\}$ be a disjoint family of similarities on $D$, and let $C$ be an arbitrary subset of $D$. For each positive integer $i$

(a) $\alpha_{i}(C)=m \alpha_{i-1}(C)$,

(b) $\alpha_{i}(C)=\alpha_{i+1}\left(S_{j}(C)\right) \quad(j=1, \ldots, m)$,

(c) $\alpha_{i}(C)=\alpha_{i}\left(\bigcup_{j=1}^{m} S_{j}(C)\right)$.

Proof. (a) It follows from Definition 2 that

$$
\alpha_{i}(C)=\lim _{\ell \rightarrow \infty} \frac{m \#\left\{a \in \mathcal{I}_{i-1+\ell+1}: S_{a}(D) \subset C\right\}}{m^{\ell+1}}=m \alpha_{i-1}(C) .
$$

(b) Let $A, B$ be arbitrary subsets of $D$ and $j \in\{1, \ldots, m\}$ fixed. If $x \in A \backslash B$ and $S_{j}(A) \subset S_{j}(B)$, then there exists some $y \in B$ such that $S_{j}(y)=S_{j}(x)$ contrary to the injectivity of $S_{j}$. So $S_{j}(A) \not \subset S_{j}(B)$ if $A \not \subset B$. On the other hand, it is obvious that $S_{j}(A) \subset S_{j}(B)$ if $A \subset B$. Hence, we obtain that $S_{j}(A) \subset S_{j}(B)$ if and only if $A \subset B$. So, for all $j \in\{1, \ldots, m\}$, we have

$$
\alpha_{i}(C)=\lim _{\ell \rightarrow \infty} \frac{\#\left\{a \in \mathcal{I}_{i+\ell}: S_{j} \circ S_{a}(D) \subset S_{j}(C)\right\}}{m^{\ell}} .
$$

Consequently if $S_{a}(D) \subset C$, then $S_{j} \circ S_{a}(D) \subset S_{j}(C) \subset S_{j}(D)$ and $S_{k} \circ$ $S_{a}(D) \subset S_{k}(C) \subset S_{k}(D)$ for all $j, k \in\{1, \ldots, m\}$. If $j \neq k$, then $S_{k} \circ S_{a}(D) \not \subset$ $S_{j}(C)$, since $\left\{S_{1}, \ldots, S_{m}\right\}$ is disjoint. Thus, combining this fact with the above equality, we get

$$
\alpha_{i}(C)=\lim _{\ell \rightarrow \infty} \frac{\#\left\{a \in \mathcal{I}_{i+1+\ell}: S_{a}(D) \subset S_{j}(C)\right\}}{m^{\ell}}=\alpha_{i+1}\left(S_{j}(C)\right) .
$$

(c) Since $\left\{S_{1}, \ldots, S_{m}\right\}$ is disjoint, by $(a)$ and $(b)$, we obtain

$$
\alpha_{i}\left(\bigcup_{j=1}^{m} S_{j}(C)\right)=\sum_{j=1}^{m} \alpha_{i}\left(S_{j}(C)\right)=\sum_{j=1}^{m} \alpha_{i-1}(C)=m \alpha_{i-1}(C)=\alpha_{i}(C) .
$$




\section{Method of Substitution}

Let $D \neq \emptyset$ be a closed subset of $\mathbb{R}^{n}$ and let $\left\{S_{1}, \ldots, S_{m}\right\}$ be a disjoint family of similarities on $D$ with common ratio $c(0<c<1)$. Suppose $D_{o}$ is a subset of $D$ with non-empty interior such that $c^{i_{*}+1} d(D)<d\left(D_{o}\right) \leq c^{i_{*}} d(D)$ for a fixed non-negative integer $i_{*}$. Let $D_{v}$ be a subset of $D$ for which $c^{i_{*}+1} d(D)<$ $d\left(D_{v}\right) \leq c^{i_{*}} d(D)$, and suppose that there exist an integer $i_{v}\left(\geq i_{*}\right)$ and a finite sequence $a \in \mathcal{I}_{i_{v}}$ satisfying $S_{a}(D) \subset D_{v}$. (The last hypothesis guarantees that $\alpha_{0}\left(D_{v}\right)>0$.)

We now introduce a new method to estimate Hausdorff measures of selfsimilar sets.

(a) Let $\varepsilon>0$ be given such that $\varepsilon /\left(1-\alpha_{0}\left(D_{v}\right)\right)$ is sufficiently small (cf. the proof of Lemma 2 below). According to Definition 2, it is possible to choose a positive integer $i_{0}$ such that

$$
m^{i_{0}}\left(\alpha_{i_{*}}\left(D_{o}\right)-\varepsilon\right)<n_{0}=\#\left\{a \in \mathcal{I}_{i_{*}+i_{0}}: S_{a}(D) \subset D_{o}\right\}<m^{i_{0}}\left(\alpha_{i_{*}}\left(D_{o}\right)+\varepsilon\right) .
$$

Let $I_{0}^{1}, \ldots, I_{0}^{n_{0}}$ be an enumeration of the set $\left\{S_{a}(D): S_{a}(D) \subset D_{o} ; a \in\right.$ $\left.\mathcal{I}_{i_{*}+i_{0}}\right\}$. For every $j=1, \ldots, n_{0}$ there is exactly one $a \in \mathcal{I}_{i_{*}+i_{0}}$ with $I_{0}^{j}=$ $S_{a}(D)$. Divide each $I_{0}^{j}\left(j=1, \ldots, n_{0}\right)$ into $V_{0}^{j}=S_{a}\left(D_{v}\right)$ (where $a \in \mathcal{I}_{i_{*}+i_{0}}$ with $\left.I_{0}^{j}=S_{a}(D)\right)$ and $R_{0}^{j}=I_{0}^{j} \backslash V_{0}^{j}$.

We further describe the process of our method by induction on $\ell=0,1,2, \ldots$.

(b) Assume that we have already chosen a sufficiently large integer $i_{\ell}(\ell \geq$ 1) such that

$$
\begin{aligned}
m^{i_{\ell}} \sum_{j=1}^{n_{\ell-1}}\left(\alpha_{i_{*}+i_{0}+\cdots+i_{\ell-1}}\left(R_{\ell-1}^{j}\right)-\varepsilon\right) & <n_{\ell} \\
& <m^{i_{\ell}} \sum_{j=1}^{n_{\ell-1}}\left(\alpha_{i_{*}+i_{0}+\cdots+i_{\ell-1}}\left(R_{\ell-1}^{j}\right)+\varepsilon\right)
\end{aligned}
$$

where

$$
\begin{aligned}
n_{\ell}=\#\left\{a \in \mathcal{I}_{i_{*}+i_{0}+\cdots+i_{\ell}} \text { : there exists some } j \in\left\{1, \ldots, n_{\ell-1}\right\}\right. \\
\text { with } \left.S_{a}(D) \subset R_{\ell-1}^{j}\right\} .
\end{aligned}
$$

Then, let $I_{\ell}^{1}, \ldots, I_{\ell}^{n_{\ell}}$ be an enumeration of the set

$$
\left\{S_{a}(D): S_{a}(D) \subset \bigcup_{j=1}^{n_{\ell-1}} R_{\ell-1}^{j} ; a \in \mathcal{I}_{i_{*}+i_{0}+\cdots+i_{\ell}}\right\} .
$$


For every $j=1, \ldots, n_{\ell}$ there exists a unique $a \in \mathcal{I}_{i_{*}+i_{0}+\cdots+i_{\ell}}$ such that $I_{\ell}^{j}=S_{a}(D)$. Divide every $I_{\ell}^{j}\left(j=1, \ldots, n_{\ell}\right)$ into $V_{\ell}^{j}=S_{a}\left(D_{v}\right)$ (where $a \in \mathcal{I}_{i_{*}+i_{0}+\cdots+i_{\ell}}$ with $\left.I_{\ell}^{j}=S_{a}(D)\right)$ and $R_{\ell}^{j}=I_{\ell}^{j} \backslash V_{\ell}^{j}$. By Definition 2, we can again choose a large integer $i_{\ell+1}$ such that

$$
m^{i_{\ell+1}} \sum_{j=1}^{n_{\ell}}\left(\alpha_{i_{*}+i_{0}+\cdots+i_{\ell}}\left(R_{\ell}^{j}\right)-\varepsilon\right)<n_{\ell+1}<m^{i_{\ell+1}} \sum_{j=1}^{n_{\ell}}\left(\alpha_{i_{*}+i_{0}+\cdots+i_{\ell}}\left(R_{\ell}^{j}\right)+\varepsilon\right),
$$

where

$$
\begin{gathered}
n_{\ell+1}=\#\left\{a \in \mathcal{I}_{i_{*}+i_{0}+\cdots+i_{\ell+1}}: \text { there exists some } j \in\left\{1, \ldots, n_{\ell}\right\}\right. \\
\text { with } \left.S_{a}(D) \subset R_{\ell}^{j}\right\} .
\end{gathered}
$$

(c) Repeat the process $(b)$ for $\ell+1$.

Definition 3. Let $h \in \mathcal{H}$. Suppose $\left\{S_{1}, \ldots, S_{m}\right\}$ is a disjoint family of similarities on $D$ with common ratio $c$. The above process is called $D_{v}$-substitution of $D_{o}$ with respect to the sequence $\left(i_{\ell}\right)$. Every $V_{\ell}^{j}\left(\ell=0,1,2, \ldots ; j=1, \ldots, n_{\ell}\right)$ is called an element of $D_{v}$-substitution of $D_{o}$. The $D_{v}$-substitution of $D_{o}$ is said to be efficient if

$$
\sigma\left(D_{o} ; D_{v}\right)=\lim _{\varepsilon \rightarrow 0} \sum_{\substack{V \text { is an element of } \\ D_{v} \text {-substitution of } D_{o}}} h(d(V))<h\left(d\left(D_{o}\right)\right) .
$$

Remark 1. Obviously, $\left\{V_{\ell}^{j}\right\}$ which was obtained from the above process covers almost all of the self-similar set $F$ under $S_{1}, \ldots, S_{m}$. Indeed, for every $h \in \mathcal{H}$ we can select a covering of $F$ consisting of $\left\{V_{\ell}^{j}\right\}$ and $\left\{E_{j}\right\}$ such that the values of $\sum h\left(d\left(E_{j}\right)\right)$ and $\sum \alpha_{i_{*}}\left(E_{j}\right)$ are as small as desired by taking the values of $i_{\ell}$ 's sufficiently large in the above process.

Lemma 2. For every positive integer $\ell$

$$
\begin{aligned}
m^{i_{0}+\cdots+i_{\ell}}\left(\alpha_{i_{*}}\left(D_{o}\right)-\varepsilon\right) & \left(1-\alpha_{0}\left(D_{v}\right)-\varepsilon\right)^{\ell}<n_{\ell} \\
& <m^{i_{0}+\cdots+i_{\ell}}\left(\alpha_{i_{*}}\left(D_{o}\right)+\varepsilon\right)\left(1-\alpha_{0}\left(D_{v}\right)+\varepsilon\right)^{\ell} .
\end{aligned}
$$

Proof. By Lemma 1 (b) we have, for every positive integer $\ell$,

$$
\begin{aligned}
\alpha_{i_{*}+i_{0}+\cdots+i_{\ell-1}}\left(R_{\ell-1}^{j}\right) & =\alpha_{i_{*}+i_{0}+\cdots+i_{\ell-1}}\left(I_{\ell-1}^{j} \backslash V_{\ell-1}^{j}\right) \\
& =\alpha_{i_{*}+i_{0}+\cdots+i_{\ell-1}}\left(S_{a}(D) \backslash S_{a}\left(D_{v}\right)\right) \\
& =\alpha_{i_{*}+i_{0}+\cdots+i_{\ell-1}}\left(S_{a}(D)\right)-\alpha_{i_{*}+i_{0}+\cdots+i_{\ell-1}}\left(S_{a}\left(D_{v}\right)\right) \\
& =1-\alpha_{0}\left(D_{v}\right)
\end{aligned}
$$


where $a \in \mathcal{I}_{i_{*}+i_{0}+\cdots+i_{\ell-1}}$ with $S_{a}(D)=I_{\ell-1}^{j}$. Combining this result with the inequalities for $n_{\ell}$ 's in the above process $(b)$ and by induction on $\ell$, we complete the proof.

Theorem 3. Let $\left\{S_{1}, \ldots, S_{m}\right\}$ be a disjoint family of similarities on $D$ with common ratio c. Suppose $h \in \mathcal{H}_{m}^{c}$. If $i_{*}$ is so large that the relation (2) holds for all $0<t \leq c^{i_{*}} d(D)$, then we have $\sigma\left(D_{o} ; D_{v}\right)=\alpha_{i_{*}}\left(D_{o}\right) \frac{h\left(d\left(D_{v}\right)\right)}{\alpha_{i_{*}}\left(D_{v}\right)}$.

Proof. It follows from Definition 3, Lemma 2 and Lemma 1 (a) that

$$
\begin{aligned}
\sigma\left(D_{o} ; D_{v}\right) & =\lim _{\varepsilon \rightarrow 0} \sum_{\ell=0}^{\infty} \sum_{j=1}^{n_{\ell}} h\left(d\left(V_{\ell}^{j}\right)\right) \\
& =\lim _{\varepsilon \rightarrow 0} \sum_{\ell=0}^{\infty} n_{\ell} h\left(c^{i_{*}+i_{0}+\cdots+i_{\ell}} d\left(D_{v}\right)\right) \\
& =\lim _{\varepsilon \rightarrow 0} \sum_{\ell=0}^{\infty} \frac{n_{\ell}}{m^{i_{*}+i_{0}+\cdots+i_{\ell}}} h\left(d\left(D_{v}\right)\right) \\
& =\frac{\alpha_{i_{*}}\left(D_{o}\right)}{m^{i_{*}}} \frac{h\left(d\left(D_{v}\right)\right)}{\alpha_{0}\left(D_{v}\right)} \\
& =\alpha_{i_{*}}\left(D_{o}\right) \frac{h\left(d\left(D_{v}\right)\right)}{\alpha_{i_{*}}\left(D_{v}\right)} .
\end{aligned}
$$

Definition 4. Suppose $\left\{S_{1}, \ldots, S_{m}\right\}$ is a disjoint family of similarities on $D$ with common ratio $c$. Let $i$ be a non-negative integer. Define

$$
\Phi_{i}\left(D_{1}\right)=\inf \left\{\frac{h(d(I))}{\alpha_{i}(I)}: I \subset D_{1} ; c^{i+1} d(D)<d(I) \leq c^{i} d(D)\right\}
$$

for all subsets $D_{1}$ of $D$ with $d\left(D_{1}\right) \geq c^{i} d(D)$, where we follow the convention that if $a>0$, then $a / 0:=\infty$.

In the definition of $\Phi_{i}$, the infimum has to be taken over all 'test' sets $I$ whose diameters lie between $c^{i+1} d(D)$ and $c^{i} d(D)$. Therefore, the $\Phi_{i}$ can be defined on the only sets $D_{1}$ with $d\left(D_{1}\right) \geq c^{i} d(D)$.

Definition 5. For any compact subsets $A, B$ of $D$ the distance $\rho(A, B)$ between $A$ and $B$ is defined by $\rho(A, B)=\min \{|x-y|: x \in A ; y \in B\}$. Suppose $\left\{S_{1}, \ldots, S_{m}\right\}$ is a disjoint family of similarities on $D$ with common ratio $c$. Let $\delta=\min \left\{\rho\left(S_{i}(D), S_{j}(D)\right): i \neq j\right\}$ and $\tau=\min \left\{i \in \mathbb{N}_{0}: c^{i+1} d(D) \leq \delta\right\}$. The constant $\tau$ is called the index of the self-similar set under $S_{1}, \ldots, S_{m}$. 
Lemma 4. Let $\tau$ be the index of the self-similar set under $S_{1}, \ldots, S_{m}$ with common ratio $c$. Assume that $i \geq \tau$ and $a \in \mathcal{I}_{i-\tau}$ are fixed. Let $h \in \mathcal{H}$. Then $\Phi_{i}\left(S_{a}(D)\right)=\Phi_{i}(D)$, where we set $S_{a}(D)=D$ for $a \in \mathcal{I}_{0}$.

Proof. If $i=\tau$, the statement of the lemma is obvious. Now, let $i>\tau$, then we have by the fact that $S_{1}, \ldots, S_{m}$ are similarities with common ratio $c$ and by Definition 5

$$
\begin{aligned}
\min \left\{\rho\left(S_{b}(D), S_{b^{\prime}}(D)\right)\right. & \left.: b, b^{\prime} \in \mathcal{I}_{i-\tau} ; b \neq b^{\prime}\right\} \\
& =c^{i-\tau-1} \min \left\{\rho\left(S_{j}(D), S_{k}(D)\right): j \neq k\right\} \\
& \geq c^{i-\tau-1} c^{\tau+1} d(D) \\
& =c^{i} d(D) .
\end{aligned}
$$

For any subset $A$ of $D$ let

$$
\begin{aligned}
& \mathcal{C}_{1}(A)=\left\{I \subset D: I \subset A ; c^{i+1} d(D)<d(I) \leq c^{i} d(D)\right\}, \\
& \mathcal{C}_{2}(A)=\left\{I \subset D: I \cap A \neq \emptyset ; I \not \subset A ; c^{i+1} d(D)<d(I) \leq c^{i} d(D)\right\}
\end{aligned}
$$

and

$$
\mathcal{C}_{3}(A)=\left\{I \subset D: I \cap A=\emptyset ; c^{i+1} d(D)<d(I) \leq c^{i} d(D)\right\} .
$$

Then we have

$$
\mathcal{C}_{1}(D)=\bigcup_{b \in \mathcal{I}_{i-\tau}} \mathcal{C}_{1}\left(S_{b}(D)\right) \cup \bigcup_{b \in \mathcal{I}_{i-\tau}} \mathcal{C}_{2}\left(S_{b}(D)\right) \cup \mathcal{C}_{3}\left(\bigcup_{b \in \mathcal{I}_{i-\tau}} S_{b}(D)\right) .
$$

Since the structure in $S_{b}(D)$ is congruent to that in $S_{a}(D)$ and (3) implies that if $I \in \mathcal{C}_{2}\left(S_{b}(D)\right)$, then $\#\left(I \cap S_{b^{\prime}}(D)\right) \leq 1$ for any $b^{\prime} \in \mathcal{I}_{i-\tau}$ with $b^{\prime} \neq b$, the above equality implies that

$$
\sup \left\{\alpha_{i}(I): I \in \mathcal{C}_{1}(D)\right\}=\sup \left\{\alpha_{i}(I): I \in \mathcal{C}_{1}\left(S_{a}(D)\right)\right\}
$$

Further, since the structure in $S_{b}(D)$ is congruent to that in $S_{a}(D)$, we see that

$$
\inf \left\{\frac{h(d(I))}{\alpha_{i}(I)}: I \in \mathcal{C}_{1}\left(S_{b}(D)\right)\right\}=\inf \left\{\frac{h(d(I))}{\alpha_{i}(I)}: I \in \mathcal{C}_{1}\left(S_{a}(D)\right)\right\}
$$

for any $b \in \mathcal{I}_{i-\tau}$. As it was already stated, (3) implies that if $I \in \mathcal{C}_{2}\left(S_{b}(D)\right)$, then $\#\left(I \cap S_{b^{\prime}}(D)\right) \leq 1$ and hence $\alpha_{i}\left(I \cap S_{b^{\prime}}(D)\right)=0$ for each $b^{\prime} \in \mathcal{I}_{i-\tau}$ with $b^{\prime} \neq b$. Hence, we get

$$
\inf \left\{\frac{h(d(I))}{\alpha_{i}(I)}: I \in \mathcal{C}_{2}\left(S_{b}(D)\right)\right\} \geq \inf \left\{\frac{h(d(I))}{\alpha_{i}(I)}: I \in \mathcal{C}_{1}\left(S_{a}(D)\right)\right\} .
$$


Trivially, we have

$$
\inf \left\{\frac{h(d(I))}{\alpha_{i}(I)}: I \in \mathcal{C}_{3}\left(\bigcup_{b \in \mathcal{I}_{i-\tau}} S_{b}(D)\right)\right\}=\infty .
$$

Therefore, we may conclude that

$$
\begin{aligned}
\Phi_{i}(D) & =\inf \left\{\frac{h(d(I))}{\alpha_{i}(I)}: I \in \mathcal{C}_{1}(D)\right\} \\
& =\inf \left\{\frac{h(d(I))}{\alpha_{i}(I)}: I \in \mathcal{C}_{1}\left(S_{a}(D)\right)\right\} \\
& =\Phi_{i}\left(S_{a}(D)\right) .
\end{aligned}
$$

Lemma 5. Suppose $\left\{S_{1}, \ldots, S_{m}\right\}$ is a disjoint family of similarities on $D$ with common ratio $c$. Let $h \in \mathcal{H}_{m}^{c}$. Suppose $i_{*}$ is given such that (2) holds for all $0<t \leq c^{i_{*}} d(D)$. Assume that $D_{1}, D_{2}$ are subsets of $D$ such that

$$
\begin{aligned}
c^{i_{*}+1} d(D)<d\left(D_{1}\right) & \leq c^{i_{*}} d(D) ; c^{i_{*}+1} d(D)<d\left(D_{2}\right) \\
& \leq c^{i_{*}} d(D) ; \quad \frac{h\left(d\left(D_{1}\right)\right)}{\alpha_{i_{*}}\left(D_{1}\right)}<\frac{h\left(d\left(D_{2}\right)\right)}{\alpha_{i_{*}}\left(D_{2}\right)} .
\end{aligned}
$$

(a) There exists an efficient $D_{1}$-substitution of $D_{2}$.

(b) There exists no efficient $D_{2}$-substitution of $D_{1}$.

Proof. (a) Using Theorem 3 we obtain

$$
\sigma\left(D_{2} ; D_{1}\right)=\alpha_{i_{*}}\left(D_{2}\right) \frac{h\left(d\left(D_{1}\right)\right)}{\alpha_{i_{*}}\left(D_{1}\right)}<\alpha_{i_{*}}\left(D_{2}\right) \frac{h\left(d\left(D_{2}\right)\right)}{\alpha_{i_{*}}\left(D_{2}\right)}=h\left(d\left(D_{2}\right)\right) .
$$

(b) As in the proof of $(a)$, it is easy to see

$$
\sigma\left(D_{1} ; D_{2}\right)=\alpha_{i_{*}}\left(D_{1}\right) \frac{h\left(d\left(D_{2}\right)\right)}{\alpha_{i_{*}}\left(D_{2}\right)}>\alpha_{i_{*}}\left(D_{1}\right) \frac{h\left(d\left(D_{1}\right)\right)}{\alpha_{i_{*}}\left(D_{1}\right)}=h\left(d\left(D_{1}\right)\right)
$$

by using Theorem 3 again.

Lemma 6. Let $\tau$ be the index of the self-similar set under $S_{1}, \ldots, S_{m}$ with common ratio c. Let $h \in \mathcal{H}_{m}^{c}$. Suppose $i(\geq \tau)$ is a given positive integer such that (2) holds for all $0<t \leq c^{i-\tau} d(D)$. Then we have $m^{i} \Phi_{i}(D)=$ $m^{i+1} \Phi_{i+1}(D)$. 
Proof. By Lemma $1(a)$ and $(b)$ we obtain, for any $j \in\{1, \ldots, m\}$,

$$
\begin{aligned}
\Phi_{i}(D) & =\inf \left\{\frac{h(d(I))}{\alpha_{i}(I)}: I \subset D ; c^{i+1} d(D)<d(I) \leq c^{i} d(D)\right\} \\
& =m \cdot \inf \left\{\frac{h\left(d\left(S_{j}(I)\right)\right)}{\alpha_{i+1}\left(S_{j}(I)\right)}: I \subset D ; c^{i+1} d(D)<d(I) \leq c^{i} d(D)\right\} \\
& \geq m \cdot \inf \left\{\frac{h(d(J))}{\alpha_{i+1}(J)}: J \subset D ; c^{i+2} d(D)<d(J) \leq c^{i+1} d(D)\right\} \\
& =m \Phi_{i+1}(D),
\end{aligned}
$$

where the second equality holds because, by (2) and our hypothesis for $i$, we have

$$
h\left(d\left(S_{j}(I)\right)\right)=(1 / m) h(d(I)) \quad \text { and } \quad \alpha_{i+1}\left(S_{j}(I)\right)=\alpha_{i}(I) .
$$

On the other hand, let $I$ be a subset of $D$ with $c^{i+2} d(D)<d(I) \leq c^{i+1} d(D)$.

Case I. Assume that there exists a finite sequence $a=\left(a_{1}, \ldots, a_{i+1-\tau}\right) \in$ $\mathcal{I}_{i+1-\tau}$ such that $I \subset S_{a}(D)$. Then we may choose a subset $I^{\prime}$ of $S_{a_{2}} \circ \cdots \circ$ $S_{a_{i+1-\tau}}(D)$ such that $I=S_{a_{1}}\left(I^{\prime}\right)$. Hence, using the properties of $S_{j}$ and Lemma $1(b)$ we have $d(I)=c d\left(I^{\prime}\right)$ and $\alpha_{i+1}(I)=\alpha_{i}\left(I^{\prime}\right)$ and so, using the properties of $h$

$$
\frac{h(d(I))}{\alpha_{i+1}(I)}=\frac{1}{m} \frac{h\left(d\left(I^{\prime}\right)\right)}{\alpha_{i}\left(I^{\prime}\right)} .
$$

Case II. Now assume that every $S_{a}(D), a \in \mathcal{I}_{i+1-\tau}$, does not include $I$. In view of (3) we have

$$
\min \left\{\rho\left(S_{b}(D), S_{b^{\prime}}(D)\right): b, b^{\prime} \in \mathcal{I}_{i+1-\tau} ; b \neq b^{\prime}\right\} \geq c^{i+1} d(D) \geq d(I) .
$$

Therefore, we may merely consider the case where there exists a unique $a \in$ $\mathcal{I}_{i+1-\tau}$ such that the interior of the intersection of $I$ and $S_{a}(D)$ is non-empty. Due to the properties of the self-similar sets there exists a set $I_{1} \subset \mathbb{R}^{n}$ similar to $I$ with $\alpha_{i}\left(I_{1}\right)=\alpha_{i}\left(I_{1} \cap D\right)=\alpha_{i+1}(I)$ and $c d\left(I_{1}\right)=d(I)$. Thus, we may choose a subset $I^{\prime}$ of $D$ including $I_{1} \cap D$ and satisfying $\alpha_{i}\left(I^{\prime}\right) \geq \alpha_{i+1}(I)$ and $c d\left(I^{\prime}\right)=d(I)$. Since

$$
d\left(I_{1}\right)=c^{-1} d(I) \leq c^{i} d(D)<d(D)
$$

we may select an appropriate subset $I_{2}$ of $D$ such that $d\left(\left(I_{1} \cap D\right) \cup I_{2}\right)=d\left(I_{1}\right)$. If we put $I^{\prime}=\left(I_{1} \cap D\right) \cup I_{2}$, then $I^{\prime}$ satisfies the desired properties. Hence, using the properties of $h$

$$
\frac{h(d(I))}{\alpha_{i+1}(I)} \geq \frac{1}{m} \frac{h\left(d\left(I^{\prime}\right)\right)}{\alpha_{i}\left(I^{\prime}\right)}
$$


Finally, by (5) and (6)

$$
\begin{aligned}
\Phi_{i+1}(D) & =\inf \left\{\frac{h(d(I))}{\alpha_{i+1}(I)}: I \subset D ; c^{i+2} d(D)<d(I) \leq c^{i+1} d(D)\right\} \\
& \geq \frac{1}{m} \cdot \inf \left\{\frac{h\left(d\left(I^{\prime}\right)\right)}{\alpha_{i}\left(I^{\prime}\right)}: I^{\prime} \subset D ; c^{i+1} d(D)<d\left(I^{\prime}\right) \leq c^{i} d(D)\right\} \\
& =\frac{1}{m} \cdot \Phi_{i}(D) .
\end{aligned}
$$

The assertion of the lemma follows from (4) and the last inequality.

Lemma 7. Let $F$ be the self-similar set under $S_{1}, \ldots, S_{m}$ with common ratio $c$ and let $\tau$ be the index of $F$. Let $h \in \mathcal{H}_{m}^{c}$. Suppose $i(>2 \tau)$ is a given integer such that (2) holds for all $0<t \leq c^{i-2 \tau} d(D)$. Then for any $a \in \mathcal{I}_{i-\tau}$

$$
\Phi_{i-\tau}(D)=\inf \left\{\sum_{j} h\left(d\left(U_{j}\right)\right):\left\{U_{j}\right\} \text { is a } c^{i} d(D) \text {-covering of } F \cap S_{a}(D)\right\} \text {. }
$$

Proof. Let $\varepsilon>0$ be arbitrarily small, and let $\mathcal{U}_{i}$ be the set of all $c^{i} d(D)$ coverings of $F \cap S_{a}(D)$. By Definition 4, we can choose a subset $D^{\prime}$ of $D$ such that

$$
c^{i-\tau+1} d(D)<d\left(D^{\prime}\right) \leq c^{i-\tau} d(D)
$$

and

$$
\Phi_{i-\tau}(D) \leq \frac{h\left(d\left(D^{\prime}\right)\right)}{\alpha_{i-\tau}\left(D^{\prime}\right)} \leq \Phi_{i-\tau}(D)+\varepsilon .
$$

Let $\mathcal{U}_{i}^{\varepsilon}$ be the set of all $c^{i} d(D)$-coverings of $F \cap S_{a}(D)$ consisting of $\left\{U_{j}\right\}$ and $\left\{V_{j}\right\}$ with the properties

(i) if $c^{k+1} d(D)<d\left(U_{j}\right) \leq c^{k} d(D)$, then there exists some $b \in \mathcal{I}_{k-i+\tau}$ such that

$$
\frac{h\left(d\left(U_{j}\right)\right)}{\alpha_{k}\left(U_{j}\right)} \leq \frac{h\left(d\left(S_{b}\left(D^{\prime}\right)\right)\right)}{\alpha_{k}\left(S_{b}\left(D^{\prime}\right)\right)}
$$

(ii) $\left\{V_{j}\right\}$ satisfies

$$
\sum_{j} h\left(d\left(V_{j}\right)\right)<\varepsilon ; \quad \sum_{j} \alpha_{i-\tau}\left(V_{j}\right)<\varepsilon .
$$

Now assume $\left\{B_{j}\right\} \in \mathcal{U}_{i}$ such that there exists a $j_{0}$ satisfying

$$
\frac{h\left(d\left(B_{j_{0}}\right)\right)}{\alpha_{k}\left(B_{j_{0}}\right)}>\frac{h\left(d\left(S_{b}\left(D^{\prime}\right)\right)\right)}{\alpha_{k}\left(S_{b}\left(D^{\prime}\right)\right)}
$$


for some $b \in \mathcal{I}_{k-i+\tau}$ with $c^{k+1} d(D)<d\left(B_{j_{0}}\right) \leq c^{k} d(D)$. Then by taking $i_{*}=k$ in Lemma $5(a)$, there exists an efficient $S_{b}\left(D^{\prime}\right)$-substitution $\left\{B_{j}^{\prime}\right\}$ of $B_{j_{0}} \cap D$. In particular, we may choose a $c^{i} d(D)$-covering $\left\{E_{j}\right\}$ of $\left(F \cap S_{a}(D) \cap B_{j_{0}}\right) \backslash \bigcup B_{j}^{\prime}$ such that $\sum h\left(d\left(E_{j}\right)\right)<\varepsilon / 2^{j_{0}}$ and $\sum \alpha_{i-\tau}\left(E_{j}\right)<\varepsilon / 2^{j_{0}}$ (cf. Remark 1). We now replace the $B_{j_{0}}$ in the covering $\left\{B_{j}\right\}$ by $\left\{B_{j}^{\prime}\right\} \cup\left\{E_{j}\right\}$.

As described above, we may ultimately transform the given covering $\left\{B_{j}\right\} \in$ $\mathcal{U}_{i}$ into some $\left\{U_{j}\right\} \cup\left\{V_{j}\right\} \in \mathcal{U}_{i}^{\varepsilon}$. Therefore, we have

$$
\begin{aligned}
\inf \left\{\sum_{j} h\left(d\left(U_{j}\right)\right):\left\{U_{j}\right\} \cup\left\{V_{j}\right\} \in \mathcal{U}_{i}^{\varepsilon}\right\} \\
\quad \leq \inf \left\{\sum_{j} h\left(d\left(U_{j}\right)\right):\left\{U_{j}\right\} \in \mathcal{U}_{i}\right\} \\
\quad \leq \inf \left\{\sum_{j} h\left(d\left(U_{j}\right)\right)+\sum_{j} h\left(d\left(V_{j}\right)\right):\left\{U_{j}\right\} \cup\left\{V_{j}\right\} \in \mathcal{U}_{i}^{\varepsilon}\right\} .
\end{aligned}
$$

The first inequality in (10) follows from the above consideration, and the fact $\mathcal{U}_{i}^{\varepsilon} \subset \mathcal{U}_{i}$ implies the second inequality in (10).

Now, let $\left\{U_{j}\right\} \cup\left\{V_{j}\right\} \in \mathcal{U}_{i}^{\varepsilon}$ be given such that there exists a sequence $\left(k_{j}\right)$, $k_{j} \geq i$, satisfying $c^{k_{j}+1} d(D)<d\left(U_{j}\right) \leq c^{k_{j}} d(D)$. By (8), Lemma $1(a)$ and $(7)$ in order, we have for some $b \in \mathcal{I}_{k_{j}-i+\tau}$

$$
\begin{aligned}
h\left(d\left(U_{j}\right)\right) & \leq \alpha_{k_{j}}\left(U_{j}\right) \frac{h\left(d\left(S_{b}\left(D^{\prime}\right)\right)\right)}{\alpha_{k_{j}}\left(S_{b}\left(D^{\prime}\right)\right)} \\
& =\alpha_{i-\tau}\left(U_{j}\right) \frac{h\left(d\left(S_{b}\left(D^{\prime}\right)\right)\right)}{\alpha_{i-\tau}\left(S_{b}\left(D^{\prime}\right)\right)} \\
& =\alpha_{i-\tau}\left(U_{j}\right) \frac{h\left(d\left(D^{\prime}\right)\right)}{\alpha_{i-\tau}\left(D^{\prime}\right)} \\
& \leq \alpha_{i-\tau}\left(U_{j}\right)\left(\Phi_{i-\tau}(D)+\varepsilon\right) .
\end{aligned}
$$

Since $\inf \left\{\sum \alpha_{i-\tau}\left(U_{j}\right):\left\{U_{j}\right\} \cup\left\{V_{j}\right\} \in \mathcal{U}_{i}^{\varepsilon}\right\} \leq 1$, condition (9), together with the above inequality, implies

$$
\begin{aligned}
& \inf \left\{\sum_{j} h\left(d\left(U_{j}\right)\right)+\sum_{j} h\left(d\left(V_{j}\right)\right):\left\{U_{j}\right\} \cup\left\{V_{j}\right\} \in \mathcal{U}_{i}^{\varepsilon}\right\} \\
& \quad \leq \inf \left\{\sum_{j} \alpha_{i-\tau}\left(U_{j}\right):\left\{U_{j}\right\} \cup\left\{V_{j}\right\} \in \mathcal{U}_{i}^{\varepsilon}\right\} \cdot\left(\Phi_{i-\tau}(D)+\varepsilon\right)+\varepsilon \\
& \quad \leq \Phi_{i-\tau}(D)+2 \varepsilon
\end{aligned}
$$


Since $\varepsilon>0$ may be sufficiently small, we obtain

$$
\inf \left\{\sum_{j} h\left(d\left(U_{j}\right)\right)+\sum_{j} h\left(d\left(V_{j}\right)\right):\left\{U_{j}\right\} \cup\left\{V_{j}\right\} \in \mathcal{U}_{i}^{\varepsilon}\right\} \leq \Phi_{i-\tau}(D) .
$$

On the other hand, if $\left\{U_{j}\right\} \cup\left\{V_{j}\right\}$ belongs to $\mathcal{U}_{i}^{\varepsilon}$, then for some $k$ with $c^{k+1} d(D)<d\left(U_{j}\right) \leq c^{k} d(D)$

$$
\frac{h\left(d\left(U_{j}\right)\right)}{\alpha_{k}\left(U_{j}\right)} \geq \Phi_{k}(D) .
$$

Thus, it follows from (12), Lemma $1(a)$ and Lemma 6 that

$$
h\left(d\left(U_{j}\right)\right) \geq \alpha_{k}\left(U_{j}\right) \Phi_{k}(D)=\alpha_{i-\tau}\left(U_{j}\right) \Phi_{i-\tau}(D) .
$$

Hence, as $\sum \alpha_{i-\tau}\left(U_{j}\right) \geq 1-\varepsilon$ by (9), we get

$$
\sum_{j} h\left(d\left(U_{j}\right)\right) \geq \Phi_{i-\tau}(D) \sum_{j} \alpha_{i-\tau}\left(U_{j}\right) \geq \Phi_{i-\tau}(D)(1-\varepsilon)
$$

and so

$$
\inf \left\{\sum_{j} h\left(d\left(U_{j}\right)\right):\left\{U_{j}\right\} \cup\left\{V_{j}\right\} \in \mathcal{U}_{i}^{\varepsilon}\right\} \geq \Phi_{i-\tau}(D) .
$$

The assertion of the lemma follows from (10), (11) and (13).

Now, we shall prove the main result of this paper.

Theorem 8. Suppose $F$ is the self-similar set under $S_{1}, \ldots, S_{m}$ with common ratio $c$ and let $\tau$ be the index of $F$. Let $h \in \mathcal{H}_{m}^{c}$. Suppose $i(\geq \tau)$ is a positive integer such that (2) holds for all $0<t \leq c^{i-\tau} d(D)$. Then for any $a \in \mathcal{I}_{i-\tau}$ $\mu^{h}(F)=m^{i} \Phi_{i}\left(S_{a}(D)\right)$.

Proof. Let $j(>2 \tau)$ be a sufficiently large integer for which (2) holds for all $0<t \leq c^{j-2 \tau} d(D)$. By (3), we get

$$
\min \left\{\rho\left(S_{b}(D), S_{b^{\prime}}(D)\right): b, b^{\prime} \in \mathcal{I}_{j-\tau} ; b \neq b^{\prime}\right\} \geq c^{j} d(D) .
$$

Hence, by Lemma 7, we have

$$
\begin{aligned}
\inf \{ & \left.\sum_{k} h\left(d\left(U_{k}\right)\right):\left\{U_{k}\right\} \text { is a } c^{j} d(D) \text {-covering of } F\right\} \\
& =\sum_{b \in \mathcal{I}_{j-\tau}} \inf \left\{\sum_{k} h\left(d\left(U_{k}\right)\right):\left\{U_{k}\right\} \text { is a } c^{j} d(D) \text {-covering of } F \cap S_{b}(D)\right\} \\
& =m^{j-\tau} \Phi_{j-\tau}(D) .
\end{aligned}
$$


Thus, it follows from Lemma 6 and Lemma 4 that

$$
\begin{aligned}
\mu^{h}(F) & =\lim _{j \rightarrow \infty} \inf \left\{\sum_{k} h\left(d\left(U_{k}\right)\right):\left\{U_{k}\right\} \text { is a } c^{j} d(D) \text {-covering of } F\right\} \\
& =\lim _{j \rightarrow \infty} m^{j-\tau} \Phi_{j-\tau}(D) \\
& =m^{i} \Phi_{i}(D) \\
& =m^{i} \Phi_{i}\left(S_{a}(D)\right) .
\end{aligned}
$$

\section{Applications}

First, we introduce the definition of the uniform Cantor set. Let $m(\geq 2)$ be a given integer, and we choose positive real numbers $c$ and $d$ such that $m c+(m-1) d=1$. In this section, let $D=[0,1]$ and define the similarities $S_{i}: D \rightarrow D(i=1, \ldots, m)$ by $S_{i}(x)=(i-1)(c+d)+c x$. Then $c$ is the common ratio of the similarities $S_{1}, \ldots, S_{m}$ and the family of those similarities is disjoint. The self-similar set $F$ under the $S_{i}$ 's is called a uniform Cantor set.

Let $C$ be a compact subset of $\mathbb{R}$ and let $t>0$ be given. The $t$-entropy of $C$ is defined by

$$
E(C, t)=\min \left\{n \in \mathbb{N}:\left\{U_{1}, \ldots, U_{n}\right\} \text { is a } t \text {-covering of } C\right\}
$$

Mycielski [8] and Kahnert [7] have considered the Hausdorff function

$$
h_{C}(t)= \begin{cases}1 / E(C, t) & \text { for } t>0 \\ 0 & \text { for } t=0\end{cases}
$$

to construct an invariant Hausdorff measure $\mu^{h_{C}}$. From now on, we write $\mu^{C}$ instead of $\mu^{h_{C}}$. For some interesting properties of such a measure. See $[3,4$, $8,9]$.

It is easy to see $\mu^{D}(F)=0$. Obviously, the Cantor set $F$ can be covered by $m^{n}$ intervals of the length $c^{n}$ for every positive integer $n$. Since $h_{D}\left(c^{n}\right) \leq c^{n}$, (1) implies $\mu^{D}(F) \leq \lim _{n \rightarrow \infty} m^{n} c^{n}=0$.

Analogously, we can easily prove $\mu^{F}(D)=\infty$ : Let $\left\{U_{i}\right\}$ be a $c^{n}$-covering of $D$. Then $\sum d\left(U_{i}\right) \geq 1$. Let $n_{i}(\geq n)$ be an integer with $c^{n_{i}+1}<d\left(U_{i}\right) \leq c^{n_{i}}$ for every $i$. Then

$$
h_{F}\left(d\left(U_{i}\right)\right) \geq m^{-\left(n_{i}+1\right)}=c^{n_{i}} m^{-1}(m c)^{-n_{i}}>d\left(U_{i}\right) m^{-1}(m c)^{-n}
$$


and hence

$$
\sum_{i} h_{F}\left(d\left(U_{i}\right)\right) \geq m^{-1}(m c)^{-n} \sum_{i} d\left(U_{i}\right) \geq m^{-1}(m c)^{-n} .
$$

Therefore, (1) implies $\mu^{F}(D) \geq \lim _{n \rightarrow \infty} m^{-1}(m c)^{-n}=\infty$.

Similarly, we can show $\mu^{D}(D)=1$ and $\mu^{F}(F) \leq 1$, but it is virtually impossible to determine the exact value of $\mu^{F}(F)$ by using formula (1). However, we can use Theorem 8 to evaluate the lower and upper bounds for the set $\left\{\mu^{F}(F): F\right.$ is a uniform Cantor set $\}$ as well as the exact values of $\mu^{F}(F)$ for many special cases. Moreover, Theorem 8 might provide us with a possibility to evaluate the value of $\mu^{F}(F)$ within a given error.

Remark 2. Let $C$ be a compact subset of $\mathbb{R}$ with a positive Lebesgue measure. Then we can also prove that $\mu^{C}(C)=1$.

In the following lemma, we prove $h_{F} \in \mathcal{H}_{m}^{c}$.

Lemma 9. $h_{F} \in \mathcal{H}_{m}^{c}$.

Proof. Let $n=E(F, t)$. Suppose $\left\{U_{i}\right\}_{i=1, \ldots, n}$ is a $t$-covering of $F$. Then $\left\{S_{j}\left(U_{i}\right)\right\}_{i=1, \ldots, n}$ is a $c t$-covering of $F \cap S_{j}(D)$ for any $j=1, \ldots, m$. Hence, we obtain $E(F, c t) \leq m E(F, t)$.

On the other hand, let $n=E\left(F \cap S_{j}(D), c t\right)$ and suppose $\left\{U_{i}\right\}_{i=1, \ldots, n}$ is a ct-covering of $F \cap S_{j}(D)$ for some $j=1, \ldots, m$. Then $\left\{S_{j}^{-1}\left(U_{i}\right)\right\}_{i=1, \ldots, n}$ is a $t$-covering of $F$. Hence, if $0<t<d / c$, then since $c t<d$

$$
E(F, t) \leq E\left(F \cap S_{j}(D), c t\right)=\frac{1}{m} E(F, c t) .
$$

Let $\tau$ be the index of $F$. According to Theorem 8 and Lemma 9, the value of $\mu^{F}(F)$ can be evaluated by the formula

$$
\mu^{F}(F)=m^{i} \cdot \inf \left\{\frac{h_{F}(d(I))}{\alpha_{i}(I)}: I \subset\left[0, c^{i-\tau}\right] ; c^{i+1}<d(I) \leq c^{i}\right\}
$$

where $i(>\tau)$ is a sufficiently large integer.

Theorem 10. For any uniform Cantor set $F 1 / 2 \leq \mu^{F}(F)<1$.

Proof. (a) First, assume that $I\left(\subset\left[0, c^{i-\tau}\right]\right)$ is an interval with

$$
(k+1) c^{i+1}+k c^{i} d<d(I) \leq(k+1)\left(c^{i+1}+c^{i} d\right)
$$


for some $k \in\{0,1, \ldots, m-2\}$. By considering the structure of $F$ and the fact $E\left(S_{a}(D), d(I)\right) \leq[(m+k) /(k+1)]\left(a \in \mathcal{I}_{i}\right)$, where $[x]$ denotes the greatest integer which does not exceed $x$, we conclude

$$
E(F, d(I)) \leq m^{i}\left[\frac{m+k}{k+1}\right] \quad \text { and } \quad \alpha_{i}(I) \leq \frac{k+1}{m} .
$$

Hence, the fact $m \leq(k+1)[(m+k) /(k+1)] \leq m+k \leq 2 m-2$ implies

$$
m^{i} \frac{h_{F}(d(I))}{\alpha_{i}(I)} \geq \frac{m}{(k+1)[(m+k) /(k+1)]}>\frac{1}{2} .
$$

Now, assume that $I\left(\subset\left[0, c^{i-\tau}\right]\right)$ is an interval with

$$
k\left(c^{i+1}+c^{i} d\right)<d(I) \leq(k+1) c^{i+1}+k c^{i} d
$$

for some $k \in\{1,2, \ldots, m-1\}$. As in the previous case, we obtain

$$
E(F, d(I)) \leq m^{i}\left[\frac{m+k-1}{k}\right], \quad \alpha_{i}(I) \leq \frac{k+1}{m},
$$

and hence the inequality

$$
m^{i} \frac{h_{F}(d(I))}{\alpha_{i}(I)} \geq \frac{m}{(k+1)[(m+k-1) / k]} \geq \frac{1}{2}
$$

follows from the fact $\frac{m+k-1}{k} \leq \frac{2 m}{k+1}$ which can be easily proved under the assumption $m \geq k+1$.

Altogether, Theorem 8, together with (15) and (16), implies $\mu^{F}(F) \geq 1 / 2$.

(b) Consider an interval $I=\left[0, c^{i}-c^{2 i}\right]$. By (3) we have

$$
\min \left\{\rho\left(S_{b}(D), S_{b^{\prime}}(D)\right): b, b^{\prime} \in \mathcal{I}_{i-\tau} ; b \neq b^{\prime}\right\} \geq c^{i} .
$$

Therefore, if $a \in \mathcal{I}_{i-\tau}$, then

$$
E(F, d(I))=m^{i-\tau} E\left(F \cap S_{a}(D), d(I)\right) \geq m^{i-\tau}\left(m^{\tau}+1\right)
$$

and

$$
\alpha_{i}(I)=\frac{m^{i}-1}{m^{i}} .
$$

Hence, by (14), (17) and (18), we obtain

$$
\mu^{F}(F) \leq m^{i} \frac{h_{F}(d(I))}{\alpha_{i}(I)} \leq \frac{m^{\tau}}{m^{\tau}+1} \frac{m^{i}}{m^{i}-1}<1
$$

because $i$ can be arbitrarily large.

As we can see in the following theorem, $1 / 2$ is the best possible estimation of the lower bound for $\mu^{F}(F)$ in Theorem 10 . 
Theorem 11. Let $\tau$ be the index of the uniform Cantor set $F$.

(a) If $\tau=0$, then $\mu^{F}(F)=1 / 2$.

(b) $\mu^{F}(F) \rightarrow 1$ as $\tau \rightarrow \infty$.

Proof. (a) Let $i, k$ be positive integers. Choose an interval $I=\left[0, c^{i}-c^{i+k}\right]$. Clearly, we obtain $h_{F}(d(I))=\frac{1}{2 m^{i}}, \alpha_{i}(I)=1-\frac{1}{m^{k}}$ and hence

$$
m^{i} \frac{h_{F}(d(I))}{\alpha_{i}(I)}=\frac{1}{2} \frac{m^{k}}{m^{k}-1} .
$$

By letting $k \rightarrow \infty$ in (19) and considering (14) and Theorem 10, we conclude $\mu^{F}(F)=1 / 2$.

(b) Let $\tau$ and $i(>\tau)$ be sufficiently large integers. Suppose $I\left(\subset\left[0, c^{i-\tau}\right]\right)$ is an interval for which there exists an $n \in \mathbb{N} \cup\{\infty\}$ such that $\ell_{n}\left(k_{j}\right)-c^{i+n-1} d \leq$ $d(I) \leq \ell_{n}\left(k_{j}\right)$ with

$$
\ell_{n}\left(k_{j}\right)=\sum_{j=1}^{\infty} k_{j}\left(c^{i+j}+c^{i+j-1} d\right)
$$

where $k_{j} \in\{0,1, \ldots, m-1\}, k_{1} k_{n} \neq 0$ and $k_{j}=0$ for any $j>n$ (for the case of $n \in \mathbb{N}$ ) and we follow the convention $c^{i+\infty-1}=0$ (for $n=\infty$ ). In view of (3) we have

$$
\begin{gathered}
E(F, d(I)) \leq m^{i-\tau}\left(\frac{c^{i-\tau}}{\ell_{n}\left(k_{j}\right)-c^{i+n-1} d}+1\right) \leq m^{i-\tau} \frac{c^{-\tau}+1}{k_{1} c+k_{2} c^{2}+\cdots}, \\
\alpha_{i}(I) \leq \frac{k_{1}}{m}+\frac{k_{2}}{m^{2}}+\cdots
\end{gathered}
$$

and hence

$$
\begin{aligned}
m^{i} \frac{h_{F}(d(I))}{\alpha_{i}(I)} & \geq \frac{(m c)^{\tau}\left(k_{1} c+k_{2} c^{2}+\cdots\right)}{1+c^{\tau}}\left(\frac{k_{1}}{m}+\frac{k_{2}}{m^{2}}+\cdots\right)^{-1} \\
& \geq \frac{(m c)^{\tau}}{1+c^{\tau}} g(\tau)
\end{aligned}
$$

where $g(\tau) \rightarrow 1$ as $\tau \rightarrow \infty(c \rightarrow 1 / m$ as $\tau \rightarrow \infty)$. By (14) we get

$$
\mu^{F}(F) \geq \frac{(m c)^{\tau}}{1+c^{\tau}} g(\tau)
$$

From $m c+(m-1) d=1$ and $c^{\tau+1} \leq d<c^{\tau}$ (see Definition 5) it follows that $(m c)^{\tau} \rightarrow 1$ as $\tau \rightarrow \infty$. Consequently, by Theorem 10 and (20), we conclude that $\mu^{F}(F) \rightarrow 1$ as $\tau \rightarrow \infty$.

Acknowledgment. The author would like to thank the referee for his/her careful reading of early versions of this paper and valuable suggestions. 


\section{References}

[1] E. Best, On sets of fractal dimension III, Proc. London Math. Soc. (2) 47 (1942), 436-454.

[2] K. J. Falconer, Fractal geometry, John Wiley \& Sons N.Y. 1990.

[3] J. W. Fickett and J. Mycielski, A problem of invariance for Lebesgue measure, Colloq. Math. 42 (1979), 123-125.

[4] J. W. Fickett, Approximate isometries on bounded sets with an application to measure theory, Studia Math. 72 (1981), 37-46.

[5] A. Gierl, Über das Hausdorffsche Maß gewisser Punktmengen in der Zifferntheorie, J. Reine Angew. Math. 202 (1959), 183-195.

[6] J. E. Hutchinson, Fractals and self-similarity, Indiana Univ. Math. J. 30 (1981), 713-747.

[7] D. Kahnert, Haar-Mass und Hausdorff-Mass, Lecture Notes in Math. 541, Springer 1976, 13-23.

[8] J. Mycielski, Remarks on invariant measures in metric spaces, Colloq. Math. 32 (1974), 109-116.

[9] J. Mycielski, A conjecture of Ulam on the invariance of measure in Hilbert's cube, Studia Math. 60 (1977), 1-10.

[10] C. A. Rogers, Hausdorff measures, Cambridge Univ. Press 1970. 
